Historic, Archive Document

Do not assume content reflects current scientific knowledge, policies, or practices. 



\section{ROSE BUSHES SAFE TO PLANT}

Hybrid Perpetuals Blooming Principally in June 75 c. Each. $\$ 7.50$ per Doz.

Frau Karl Durschki.,......................................... White Clio ............................................................ Blush Pink Mrs. John Laing................................................... Soft Pink Paul Neyron ..........................................................Rose General Jacquenimot ............................................mson George Dickson....................................Deep Crimson

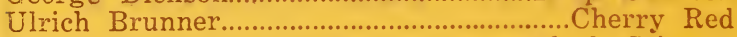
Prince Camille De Rohan.......................Black Crimson

\section{Hybrid Tea's, Blooming Continuously}

75c. Each, $\$ 7.50$ per Doz.

Kaiserin Augusta Victoria.

White White Naman Cochit. White Lady Ursula Flesh Pink Florence Pemberton....................................Flesh Pink Maman Cochet...............................................ight Pink Lady Ashtown.....................................Glistening Pink Caroline Testout..........................................Rose Pink Miss Cynthia Ford.........................................Rose Pink Laurent Carl............................................Brilliant Carmine Red Radiance..................................................Deep Red Gen. McArthur................................................Crimson Hoosier Beauty................................Glowing Crimson Gruss and Teplitz...............................Bright Crimson Hadley..................................... Deep Purplish Crimson Mrs. Leon Paine.............Silvery Flesh, Center Yellow Ophelia...............................Salmon Pink, Yellow Rose Los Angeles........................Flame Pink, Yellow Rose Lady Hillingdon...................................Apricot Yellow Mrs. Aaron Ward...................................Apricot Yellow Mrs. A. R. Waddell...............................................Salmon Sunburst ................................................ Orange Yellow

\section{Rugosa Roses}

Sir Thomas Liptorf:................................Double, White Conrad F. Meyer..........................Double, Silvery Rose Hansa..................................... Double, Reddish Violet F. J. Grootenhorst................................Double, Scarlet 
Rambler White Dorothy Perkins......................... White

Ramblex Dorothy Perkins........................................Pink

Rambler Excelsa .......................................................Red

Semi Double American Pillow......Pink, White Collar

Very Double Dr. Van Vlest..........................Blush Pink

Very Double American Beauty................... Deep Rose

Very Double Paul's Scarlet.............................Crimson

\section{Polyanthus Rose-Baby Rambler}

Gruss \& Auchin . 75c. Each. $\$ 7.50$ per Doz.

Cecil Brunner.

Orange

Baby Rambler

Pink

Lafayette

Crimson

Mrs. Wm. Koenig Red

Ellen Paulson White Pink

\section{New Hybrid Tea Roses}

Rose Marie $\$ 1.50$ Each. $\$ 15.00$ per Doz.

Souv. De Claudius Pernet. Pure Yellow

Sensation Scarlet

Edel

White

Golden Emblem

Golden

Golden Ophelia

Golden

Louise $\mathrm{K}$. Bresiau

Copper

Lolita Armour

Apricot

Mme. Butterfly

Pink

Imperial Potentate

Red

\section{New Climbers}

Dr. Huey $\$ 1.50$ Each

Chrislane De Felgonde

Gardenia

Velvety Crimson

Emily Gray

Orange

\section{Species}

Hugonis $\$ 1.00$ Each

Yellow Red

Harrison

Austrian

Yellow Persian
Yellow 
